\title{
Article \\ A Study of an EOQ Model of Growing Items with Parabolic Dense Fuzzy Lock Demand Rate
}

\author{
Suman Maity ${ }^{1} \mathbb{D}$, Sujit Kumar De ${ }^{2}$, Madhumangal Pal ${ }^{1} \mathbb{D}$ and Sankar Prasad Mondal ${ }^{3, * \mathbb{C}}$ \\ 1 Department of Applied Mathematics with Oceanology and Computer Programming, Vidyasagar University, \\ Midnapore 721102, India; maitysuman2012@gmail.com (S.M.); mmpalvu@gmail.com (M.P.) \\ 2 Department of Mathematics, Midnapore College (Autonomous), Midnapore 721101, India; \\ skdemamo2008.com@gmail.com \\ 3 Department of Applied Science, Maulana Abul Kalam Azad University of Technology, West Bengal, \\ Haringata 741249, India \\ * Correspondence: sankar.res07@gmail.com; Tel.: +91-8617561106
}

Citation: Maity, S.; De, S.K.; Pal, M.; Mondal, S.P. A Study of an EOQ Model of Growing Items with Parabolic Dense Fuzzy Lock Demand Rate. Appl. Syst. Innov. 2021, 4, 81. https://doi.org/10.3390/asi4040081

Academic Editor: Rosa M. Rodriguez

Received: 7 September 2021

Accepted: 19 October 2021

Published: 22 October 2021

Publisher's Note: MDPI stays neutral with regard to jurisdictional claims in published maps and institutional affiliations.

Copyright: (c) 2021 by the authors. Licensee MDPI, Basel, Switzerland. This article is an open access article distributed under the terms and conditions of the Creative Commons Attribution (CC BY) license (https:// creativecommons.org/licenses/by/ $4.0 /)$.

\begin{abstract}
In this article, the parabolic dense fuzzy set is defined, and its basic arithmetic operations are studied with graphical illustration. The lock set concept is incorporated in a parabolic dense fuzzy set. Then, it is applied to the problems of fishery culture via the modeling of an economic order quantity model. Here, the fingerlings are fed to reach the ideal size to fulfill the customer's demand. The growth rate of the fingerlings is assumed as a linear function. After the sales of all fish, the pond is cleaned properly for a new cycle. Here, the model is solved in a crisp sense first. Then, we fuzzify the model considering the demand rate as a parabolic dense lock fuzzy number and obtain the result in a fuzzy environment. The main aim of our study was to find the quantity of the ordering items such that the total inventory cost gets a minimum value. Lastly, sensitivity analysis and graphical illustrations were added for better justification of our model.
\end{abstract}

Keywords: inventory management; growing items; parabolic dense fuzzy number; arithmetic operations; fishery; optimization

\section{Introduction}

\subsection{Literature Review on Dense Fuzzy Sets}

Zadeh [1] first observed that there is nonrandom uncertainty behind every fact in reality. This type of uncertainty occurs not only in mathematics but also in any other research field. He handled this type of uncertainty by introducing a fuzzy set concept where each element of a set has a membership value. After that, Bellman and Zadeh [2] utilized this new concept in decision-making problems of operations research. Dubois and Prade [3] defined for the first time some arithmetic operations over fuzzy numbers. Then, Atanassov [4] developed the intuitionistic fuzzy set concept where each set element contains membership and non-membership values. The summation of membership and non-membership values always lies between zero and one. In 1985, Kaufmann and Gupta [5] introduced some fuzzy arithmetic theory and its applications. Chutia et al. [6] defined an alternative method for finding the membership value of each element in a fuzzy set. Learning experiences play a crucial role in every inventory management problem. However, most researchers did not consider this theory for simplification of inventory problems. In 2016, De and Beg [7] defined a new type of fuzzy set, namely, the dense fuzzy set, by incorporating learning experiences in a general fuzzy set. Here, all the fuzzy set components are considered a sequence of functions that converge to a crisp value for approaching learning experiences to infinity. They also defined a new defuzzification method of triangular dense fuzzy sets. After that, De and Mahata [8] introduced the concept of a cloudy fuzzy number where each component is a function of time $t$ and, after infinity time, each component converges to a crisp value. They used the cloudy fuzzy set concept to optimize a backorder economic 
order quantity (EOQ) model. In our real-life problems, we see that every decision maker established some strategies for optimizing their profit. Keeping this in mind, De [9] first defined the dense fuzzy lock set concept where the lock means the strategy taken by the decision maker. This concept is much more helpful for any decision maker to optimize the inventory profit. Faritha [10] studied parabolic fuzzy numbers for optimizing a backorder EOQ model using the nearest interval approximation. Garg [11] defined some basic arithmetic operations over generalized parabolic fuzzy numbers utilizing the concept of the distribution function. Maity et al. [12] gave a comparative study among triangular, trapezoidal, pentagonal, hexagonal, and nonlinear heptagonal dense fuzzy sets using an application in inventory problems. Maity et al. [13] also studied cloud-type nonlinear intuitionistic dense fuzzy sets and their application in inventory control problems.

\subsection{Literature Review on Inventory Models}

Harris [14] first proposed the EOQ inventory model. In this model, he minimized the total inventory cost, including the ordering cost and holding cost. After that, several researchers produced many research articles on inventory problems. All of them considered the demand rate as a deterministic one. For the first time, Karlin [15] found uncertainty in one-stage inventory problems. Today, modern researchers have shown interest in solving inventory problems in uncertain environments. De [16] studied an EOQ model considering the daytime nonrandom fuzzy demand rate. Das et al. [17] used a step order fuzzy approach for solving an EOQ model. He regarded it as uncertain daytime demand, where demand in the backorder period depends on time. De et al. [18] developed an EOQ model with selling price and promotional efforts depending on demand rate. They optimized the model using the intuitionistic fuzzy technique. Kazemi et al. [19] introduced the learning effect concept for solving an EOQ model for imperfect quality items. In 2016, De and Sana [20] studied an EPQ model with uncertain lead time and demand rate. They considered all parameters of the intuitionistic fuzzy number and used the intuitionistic fuzzy aggregation Bonferroni mean for defuzzification of the model. Karmakar et al. [21,22] considered an EPQ model and used the dense fuzzy lock set concept to reduce pollution by reusing waste items of the sponge iron industry. Maity et al. [23] developed an EOQ model with dense fuzzy demand rate where two decision makers make a single decision to optimize the model. Maity et al. [24] studied an EOQ model under daytime uncertain demand rate. They introduced a computer-based algorithm and flowchart to optimize the model by updating key vectors automatically. De and Mahata [25] considered and EOQ model of imperfect-quality items with considerable discounts. They optimized the model using cloudy fuzzy numbers. Liang and Wang [26] presented an integrated decision support model for customers to buy their desired products online. Nobil et al. [27] studied a generalized EOQ model for growing items where the size and weight of the items increase over time. He also gave a case study of a poultry farm. Rezaei [28] developed an EOQ model for growing items for fish farming and poultry industries. In these inventories, the items increase during the shortage period. Zhang et al. [29] introduced an inventory model of growing items and added a constraint of carbon emissions. De et al. [30] incorporated the carbon emission issue into an EOQ model of deteriorating items under a volumetric fuzzy system. He developed a methodology for optimizing the inventory profit function. Mahata et al. [31] studied a three-echelon supply chain model in an imperfect production system. In this model, they considered inspection error, learning effect, and return policy under a fuzzy system.

\subsection{Motivation and Specific Study}

To find the novelty of this article, we have to find the research gap first. The research gaps in some recent major studies are given in Table 1. 
Table 1. Literature review of recent major studies of growing items.

\begin{tabular}{|c|c|c|c|c|c|}
\hline \multirow{2}{*}{ Authors } & \multicolumn{2}{|c|}{ Product } & \multirow{2}{*}{ Demand } & \multirow{2}{*}{$\begin{array}{c}\text { Matter of } \\
\text { Growing Items }\end{array}$} & \multirow{2}{*}{ Solution Approach } \\
\hline & Conventional Items & Growing Items & & & \\
\hline Harris [14] & $\checkmark$ & & Crisp & Not included & $\begin{array}{c}\text { Convex } \\
\text { Optimization }\end{array}$ \\
\hline Salameh and Jaber [32] & $\checkmark$ & & Crisp & Not included & Statistical method \\
\hline Rezaei [28] & & $\checkmark$ & Crisp & Non-specific & $\begin{array}{c}\text { Convex } \\
\text { Optimization }\end{array}$ \\
\hline Zhang et al. [29] & & $\checkmark$ & Crisp & Non-specific & $\begin{array}{c}\text { Convex } \\
\text { Optimization }\end{array}$ \\
\hline Nobil et al. [27] & & $\checkmark$ & Crisp & Poultry & $\begin{array}{c}\text { Convex } \\
\text { Optimization }\end{array}$ \\
\hline $\begin{array}{l}\text { Sebatjane and } \\
\text { Adetunji [33] }\end{array}$ & & $\checkmark$ & Crisp & Non-specific & $\begin{array}{c}\text { Convex } \\
\text { Optimization }\end{array}$ \\
\hline Wee et al. [34] & & $\checkmark$ & Crisp & Non-specific & $\begin{array}{c}\text { Convex } \\
\text { Optimization }\end{array}$ \\
\hline Alfares and Afzal [35] & & $\checkmark$ & Crisp & Non-specific & $\begin{array}{c}\text { Convex } \\
\text { Optimization }\end{array}$ \\
\hline Rana et al. [36] & & $\checkmark$ & Crisp & Non-specific & $\begin{array}{c}\text { Convex } \\
\text { Optimization }\end{array}$ \\
\hline This Paper & & $\checkmark$ & $\begin{array}{l}\text { Fuzzy (PDFS } \\
\text { and PDFLS) }\end{array}$ & Fishery & $\begin{array}{c}\text { New solution algorithm } \\
\text { via new defuzzification } \\
\text { methods }\end{array}$ \\
\hline
\end{tabular}

From the above literature review of fuzzy sets, we can see that none of the researchers defined the parabolic dense fuzzy lock set concept. To avoid complexity, most researchers considered linear membership function. However, in real life, this does not always happen. In PDFLS, we incorporate the learning experience and the nonlinearity concept. The PDFLS concept may be used for not only inventory problems but also any research problem where we use a fuzzy environment. Table 1 shows that many researchers studied growing items but none of them considered the demand rate or any other parameters as a fuzzy number. However, we all know that fuzziness plays a vital role in any inventory management problem. Recently, Choudhury and Mahata [37] developed a two-echelon supply chain model of growing items. De-la-Cruz-Márquez et al. [38] studied an inventory model for growing items. Here, the demand rate was price-sensitive under carbon emissions. After that, several researchers (Gharaei and Almehdawe [39], Mittal and Sharma [40], and Alfares and Afzal [35]) discussed inventory models of growing items in a crisp environment. None of them considered fuzziness in their models. In this article, we describe the parabolic dense fuzzy number with its some basic arithmetic operations. Then, we define a parabolic dense fuzzy lock set. In this study, we also consider an EOQ model of growing items. Here, we mainly study the fishery culture using an EOQ model. We solve the EOQ model in a crisp sense first. Then, we consider the demand rate as a parabolic dense fuzzy lock number and solve the corresponding fuzzy model. A comparative study with some existing fuzzy environments is also presented, taking the numerical result from LINGO 18.0 software. Lastly, a sensitivity analysis and graphical illustration are included for better justification of the model.

This paper is arranged as follows: in Section 2, some preliminary definitions are given. In Section 3, some basic arithmetic definitions of PDFN are presented. The crisp mathematical model is developed in Section 4. In Section 5, the corresponding fuzzy mathematical model is solved. A real case study with the numerical result and sensitivity analysis of the proposed problem is given in Section 6. Section 7 contains the graphical illustration of the proposed model. Lastly, an embracing conclusion is given in Section 8. 


\section{Preliminaries}

Definition 1. Dense fuzzy number (De and Beg [7]). Let $\widetilde{B}=b_{n}, b_{n-1}, \ldots, b_{1}, b, b_{1}^{\prime}, \ldots, b_{n-1}^{\prime}, b_{n}^{\prime}$ be a $2 n+1$ tuple fuzzy number whose components are $b_{n}=b g_{n}, b_{n-1}=b g_{n-1}, \ldots, b_{1}=$ $b g_{1}, b_{1}^{\prime}=b f_{1}, \ldots, b_{n-1}^{\prime}=b f_{n-1}, b_{n}^{\prime}=b f_{n}$, where $f_{i}$ and $g_{i}$ are Cauchy sequences. If the components $f_{i}$ and $g_{i}$ converge to 1 as $n \rightarrow \infty$, then the fuzzy number $\widetilde{B}$ will also converge to $b$. Then, the fuzzy number $\widetilde{B}=b_{n}, b_{n-1}, \ldots, b_{1}, b, b_{1}^{\prime}, \ldots, b_{n-1}^{\prime}, b_{n}^{\prime}$ will be called a dense fuzzy number.

Example 1. If $\widetilde{B}=\left\langle b\left(1-\frac{\rho}{1+n}\right), b, b\left(1+\frac{\sigma}{1+n}\right)\right\rangle$ is a triangular dense fuzzy number. Then, the membership function of $\widetilde{B}$ is given by

$$
\mu(y, n)=\left\{\begin{array}{c}
\frac{y-b\left(1-\frac{\rho}{1+n}\right)}{\frac{d_{1} \rho}{1+n}}, \text { for } b\left(1-\frac{\rho}{1+n}\right) \leq y \leq b \\
\frac{b\left(1+\frac{\sigma}{1+n}\right)-y}{\frac{d_{1} \sigma}{1+n}}, \text { for } b \leq y \leq b\left(1+\frac{\sigma}{1+n}\right) \\
0, \text { for } y<b\left(1-\frac{\rho}{1+n}\right) \text { or } b\left(1+\frac{\sigma}{1+n}\right)<y
\end{array}\right.
$$

Here, $\rho$ and $\sigma$ are fuzzy deviation parameters. The values of $\rho$ and $\sigma$ lie within $(0,1)$. When the values of $\rho$ and $\sigma$ become zero, then the triangular fuzzy number is converted to a crisp number. Two-dimensional and three-dimensional graphs of the membership function of a triangular dense fuzzy number are given in Figure 1a,b, respectively. In Figure 1b, the arrow sign indicates the convergence of triangular dense fuzzy numbers.

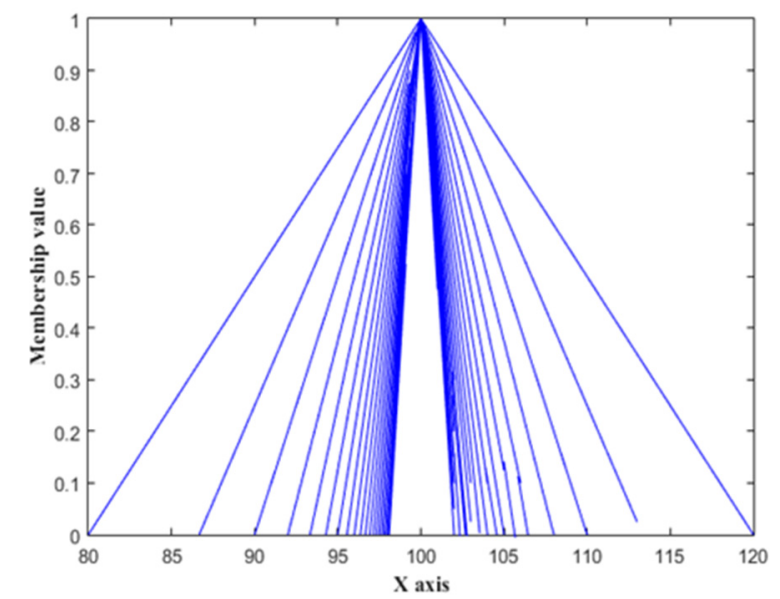

(a)

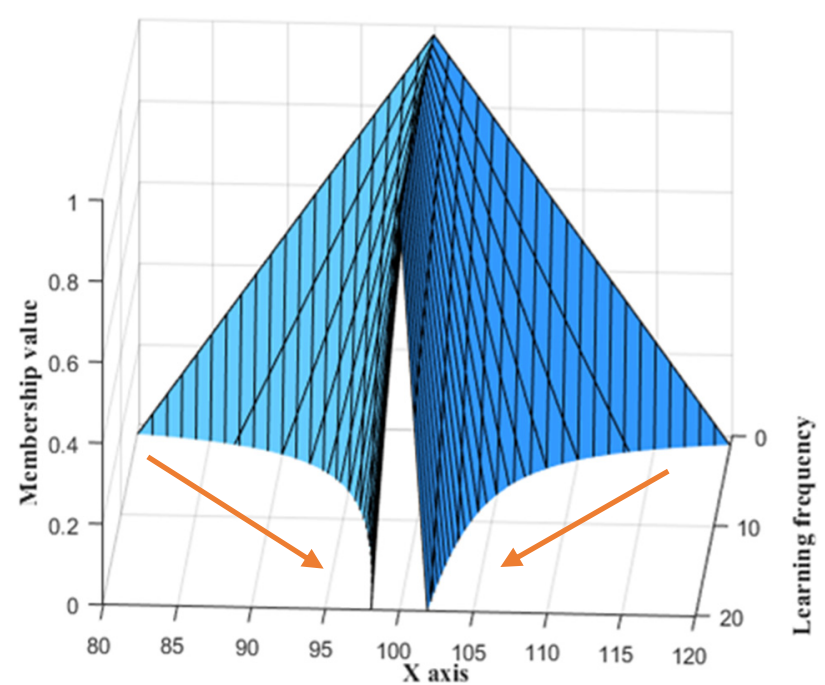

(b)

Figure 1. (a). Graph of membership function of triangular dense fuzzy number against learning frequency; (b) graph of membership function of triangular dense fuzzy number against learning frequency.

Definition 2. Parabolic fuzzy number (PFN). Garg and Ansha [11] first defined arithmetic operations on parabolic fuzzy numbers. Let $\widetilde{B}=b(1-\rho), b, b(1+\sigma)$ be a parabolic fuzzy number whose membership function is given by

$$
\mu_{B}(y)=\left\{\begin{array}{l}
\left\{\frac{y-b(1-\rho)}{b \rho}\right\}^{2}, \text { for } b(1-\rho) \leq y \leq b \\
\left\{\frac{b(1+\sigma)-y}{b \sigma}\right\}^{2}, \text { for } b \leq y \leq b(1+\sigma) \\
0, \text { for } y<b(1-\rho) \text { or } b(1+\sigma)<y
\end{array}\right.
$$

Here, $\rho$ and $\sigma$ are fuzzy deviation parameters. The values of $\rho$ and $\sigma$ lie within $(0,1)$. 
Definition 3. Parabolic dense fuzzy number (PDFN). The general form of a triangular parabolic dense fuzzy number is defined as $\widetilde{A}=\left\langle a\left(1-\frac{\rho}{1+n}\right), a, a\left(1+\frac{\sigma}{1+n}\right)\right\rangle$ associated with the membership function

$$
\mu(\widetilde{A})=\left\{\begin{array}{l}
\left\{\frac{x-a\left(1-\frac{\rho}{1+n}\right)}{\frac{a p}{1+n}}\right\}^{2}, \text { for } a\left(1-\frac{\rho}{1+n}\right) \leq x \leq a \\
\left\{\frac{a\left(1+\frac{\sigma}{1+n}\right)-x}{\frac{a \sigma}{1+n}}\right\}^{2}, \text { for } a \leq x \leq a\left(1+\frac{\sigma}{1+n}\right) \\
0, \text { for } x<a\left(1-\frac{\rho}{1+n}\right) \text { or } a\left(1+\frac{\sigma}{1+n}\right)<x
\end{array}\right.
$$

Here, the values of fuzzy deviation parameters $\rho$ and $\sigma$ lie within $(0,1)$. The plot of a triangular parabolic dense fuzzy number is given in Figure 2. This figure shows the variation in membership function with respect to learning experience.

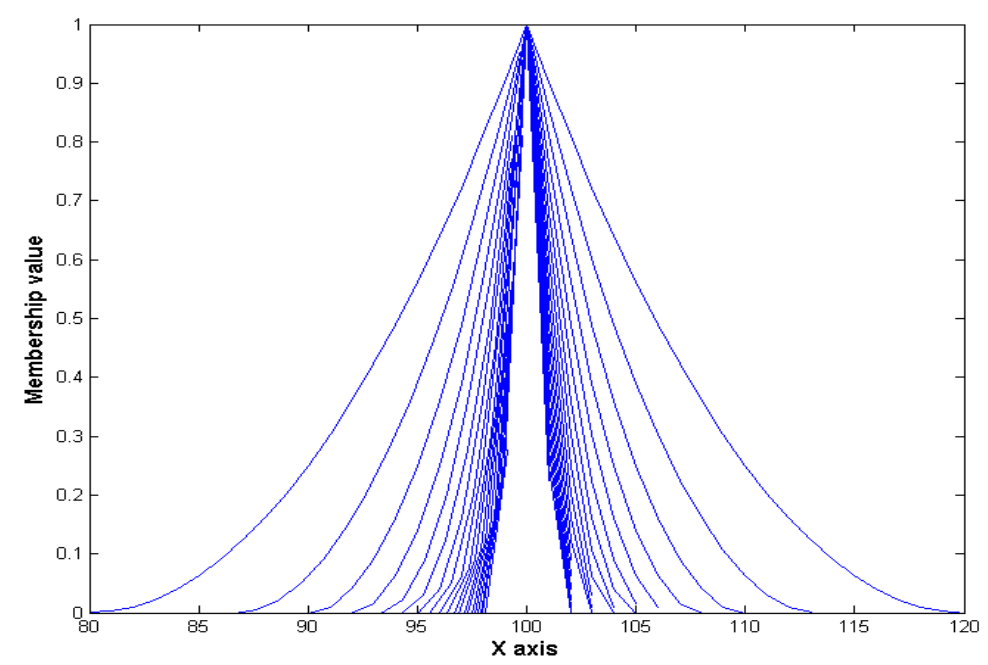

Figure 2. Graph of membership function of PDFN against learning experience.

Definition 4. Parabolic dense fuzzy lock set (PDFLS). Let $\widetilde{B}=\left\langle b\left(1-\rho u_{n}\right), b, b\left(1+\sigma v_{n}\right)\right\rangle$ for $0<\rho, \sigma<1$ be a fuzzy set, where $u_{n}$ and $v_{n}$ are Cauchy sequences. If the sequences $u_{n}$ and $v_{n}$ converge to $\frac{1}{l_{1}}$ and $\frac{1}{l_{2}} ; 0 \neq l_{1}, l_{2} \in R$, respectively, then the fuzzy set is called PDFLS and its membership function is given by

$$
\mu(\widetilde{B})=\left\{\begin{array}{r}
\left\{\frac{x-b\left(1-\rho u_{n}\right)}{b \rho u_{n}}\right\}^{2}, \text { for } b\left(1-\rho u_{n}\right) \leq x \leq b \\
\left\{\frac{b\left(1+\sigma v_{n}\right)-x}{b \sigma v_{n}}\right\}^{2}, \text { for } b \leq x \leq b\left(1+\sigma v_{n}\right) \\
0, \text { for } x<b\left(1-\rho u_{n}\right) \text { or } b\left(1+\sigma v_{n}\right)<x
\end{array}\right.
$$

Defuzzification formula of PDFLS. Let $\widetilde{A}=\left\langle a\left(1-\frac{\rho}{1+n}\right), a, a\left(1+\frac{\sigma}{1+n}\right)\right\rangle$ be a PDFLS with the membership function given in Equation (4). Then, utilizing the left and right $\alpha$-cuts of the PDFLS, we define the new defuzzified index value as follows:

$$
I(\widetilde{A})=\frac{1}{2 N} \sum_{n=1}^{N} \int_{\alpha=0}^{1}\left(\mu_{L}^{A}+\mu_{R}^{A}\right) d \alpha,
$$

where $\mu_{L}^{A}$ and $\mu_{R}^{A}$ are left and right $\alpha$-cuts, respectively.

\section{Arithmetic Operation over Parabolic Dense Fuzzy Numbers}

Let $\widetilde{A}=\left\langle a\left(1-\frac{\rho}{1+n}\right), a, a\left(1+\frac{\sigma}{1+n}\right)\right\rangle$ and $\widetilde{B}=\left\langle b\left(1-\frac{\rho^{\prime}}{1+n}\right), b, b\left(1+\frac{\sigma^{\prime}}{1+n}\right)\right\rangle$ be two parabolic dense fuzzy numbers whose membership functions are given by 


$$
\mu_{\widetilde{A}}(x)=\left\{\begin{array}{c}
\left\{\frac{x-a\left(1-\frac{\rho}{1+n}\right)}{\frac{a \rho}{1+n}}\right\}^{2}, \text { for } a\left(1-\frac{\rho}{1+n}\right) \leq x \leq a \\
\left\{\frac{a\left(1+\frac{\sigma}{1+n}\right)-x}{\frac{a n}{1+n}}\right\}^{2}, \text { for } a \leq x \leq a\left(1+\frac{\sigma}{1+n}\right) \\
0, \text { for } x<a\left(1-\frac{\rho}{1+n}\right) \text { or } a\left(1+\frac{\sigma}{1+n}\right)<x
\end{array}\right.
$$

and

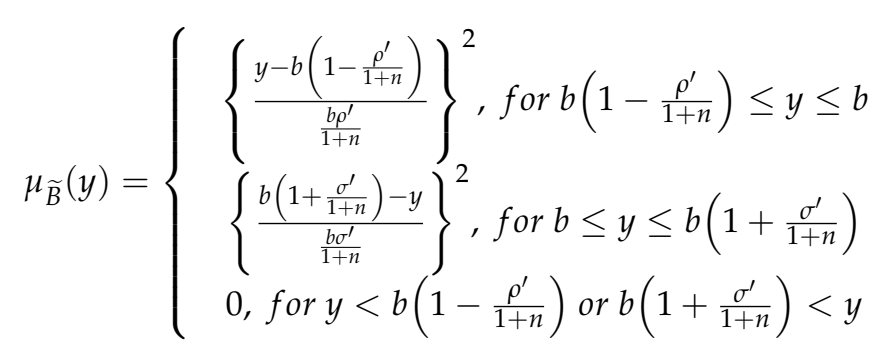

Here, $\sigma, \rho^{\prime}$, and $\sigma^{\prime}$ are fuzzy deviation parameters. The values of all the fuzzy deviation parameters lie within $(0,1)$. Now, we define some basic arithmetic operations over parabolic dense fuzzy numbers according to the extension of Garg and Ansha [11], as given below.

\section{(i) Addition of Two Parabolic Dense Fuzzy Numbers}

Let us consider two parabolic dense fuzzy numbers $\widetilde{A}$ and $\widetilde{B}$ whose membership functions are given in Equations (6) and (7). Then, the addition of these two fuzzy numbers, i.e., the fuzzy variable $\widetilde{C}=\widetilde{A}+\widetilde{B}$, is also a parabolic dense fuzzy number, and the corresponding membership function is given by

$\mu_{\widetilde{C}}(z)=\left\{\begin{aligned} & \left\{\frac{z-a\left(1-\frac{\rho}{1+n}\right)-b\left(1-\frac{\rho^{\prime}}{1+n}\right)}{\frac{a \rho}{1+n}+\frac{b \rho^{\prime}}{1+n}}\right\}^{2}, \text { for } a\left(1-\frac{\rho}{1+n}\right)+b\left(1-\frac{\rho^{\prime}}{1+n}\right) \leq z \leq a+b \\ & \left\{\frac{a\left(1+\frac{\sigma}{1+n}\right)+b\left(1+\frac{\sigma^{\prime}}{1+n}\right)-z}{\frac{a \sigma}{1+n}+\frac{b \sigma^{\prime}}{1+n}}\right\}^{2}, \text { for } a+b \leq z \leq a\left(1+\frac{\sigma}{1+n}\right)+b\left(1+\frac{\sigma^{\prime}}{1+n}\right) \\ & 0, \text { for } z<a\left(1-\frac{\rho}{1+n}\right)+b\left(1-\frac{\rho^{\prime}}{1+n}\right) \text { or } z>a\left(1+\frac{\sigma}{1+n}\right)+b\left(1+\frac{\sigma^{\prime}}{1+n}\right)\end{aligned}\right.$

Graphically, the addition of two PDFNs is given in Figure 3.

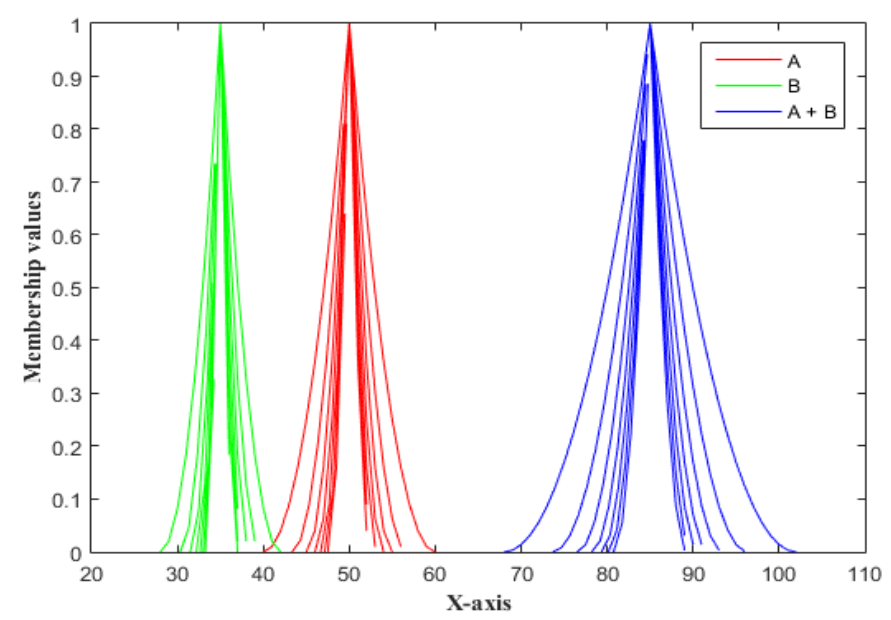

Figure 3. Addition of two parabolic dense fuzzy numbers.

\section{(ii) Subtraction of Two Parabolic Dense Fuzzy Numbers}

Let us consider two parabolic dense fuzzy numbers $\widetilde{A}$ and $\widetilde{B}$ whose membership functions are given in Equations (6) and (7). Then, the subtraction of these two fuzzy 
numbers, i.e., the fuzzy variable $\widetilde{C}=\widetilde{A}-\widetilde{B}$, is also a parabolic dense fuzzy number, and the corresponding membership function is given by

$$
\mu_{\widetilde{C}}(z)=\left\{\begin{array}{l}
\left\{\frac{z-a\left(1-\frac{\rho}{1+n}\right)+b\left(1+\frac{\sigma^{\prime}}{1+n}\right)}{\frac{a \rho}{1+n}+\frac{b \sigma^{\prime}}{1+n}}\right\}^{2}, \text { for } a\left(1-\frac{\rho}{1+n}\right)-b\left(1+\frac{\sigma^{\prime}}{1+n}\right) \leq z \leq a-b \\
\left\{\frac{a\left(1+\frac{\sigma}{1+n}\right)-b\left(1-\frac{\rho^{\prime}}{1+n}\right)-z}{\frac{a \sigma}{1+n}+\frac{b \rho^{\prime}}{1+n}}\right\}, \text { for } a-b \leq z \leq a\left(1+\frac{\sigma}{1+n}\right)-b\left(1-\frac{\rho^{\prime}}{1+n}\right) \\
0, \text { for } z<a\left(1-\frac{\rho}{1+n}\right)-b\left(1+\frac{\sigma^{\prime}}{1+n}\right) \text { or } z>a\left(1+\frac{\sigma}{1+n}\right)-b\left(1-\frac{\rho^{\prime}}{1+n}\right)
\end{array}\right.
$$

Graphically, the subtraction of two PDFNs is given in Figure 4.

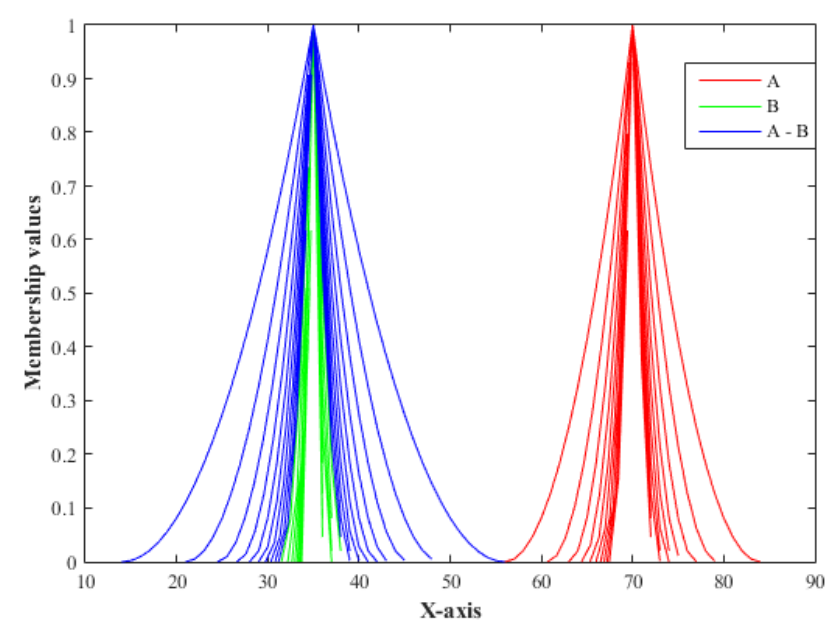

Figure 4. Subtraction of two parabolic dense fuzzy numbers.

\section{(iii) Scalar multiplication of Parabolic Dense Fuzzy Numbers}

Let $\widetilde{A}=a\left(1-\frac{\rho}{1+n}\right), a, a\left(1+\frac{\sigma}{1+n}\right)$ be a PDFN with the membership function given in Equation (6) and $z=k x$ be the transformation; then, $k \widetilde{A}$ is also a PDFN given by

$$
k \widetilde{A}=\left\{\begin{array}{l}
\left\langle k a\left(1-\frac{\rho}{1+n}\right), k a, k a\left(1+\frac{\sigma}{1+n}\right)\right\rangle \text { if } k>0 \\
\left\langle k a\left(1+\frac{\sigma}{1+n}\right), k a, k a\left(1-\frac{\rho}{1+n}\right)\right\rangle \text { if } k<0
\end{array}\right.
$$

Graphically, the scalar multiplication of two PDFNs is given in Figure 5.

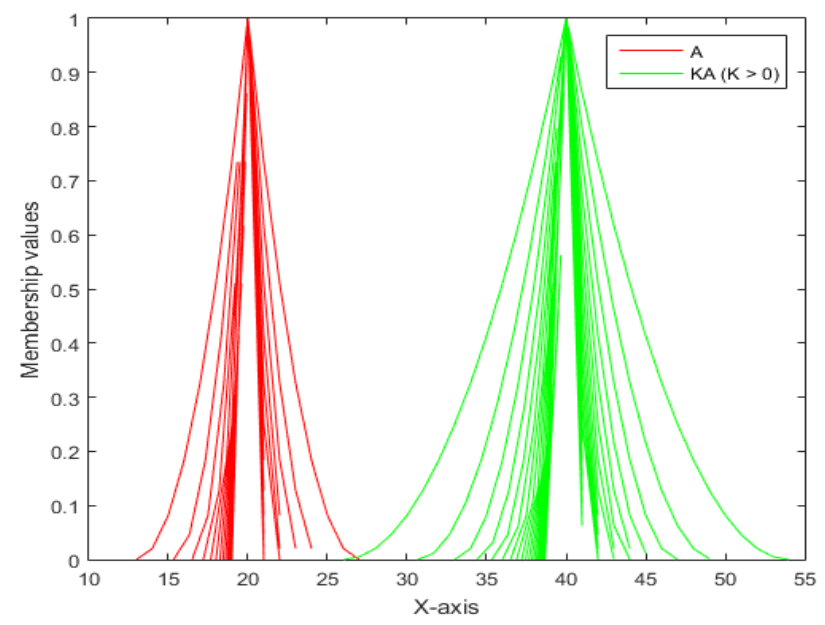

Figure 5. Scaler multiplication of two parabolic dense fuzzy numbers. 


\section{(iv) Multiplication of Two Parabolic Dense Fuzzy Numbers}

Let us consider two parabolic dense fuzzy numbers $\widetilde{A}$ and $\widetilde{B}$ whose membership functions are given in Equations (6) and (7). Then, the multiplication of these two fuzzy numbers, i.e., the fuzzy variable $\widetilde{C}=\widetilde{A} \cdot \widetilde{B}$, is also a PDFN and the corresponding membership function is given by

$$
\mu_{\widetilde{A} . \widetilde{B}}(z)=\left\{\begin{aligned}
& \left\{\frac{-Q_{1}+\sqrt{Q_{1}^{2}-4 P_{1}\left(R_{1}-z\right)}}{2 P_{1}}\right\}^{2}, \text { for } a\left(1-\frac{\rho}{1+n}\right) b\left(1-\frac{\rho^{\prime}}{1+n}\right) \leq z \leq a b \\
& \left\{\frac{-Q_{2}+\sqrt{Q_{2}^{2}-4 P_{2}\left(R_{2}-z\right)}}{2 P_{2}}\right\}^{2}, \text { for } a b \leq z \leq a\left(1+\frac{\sigma}{1+n}\right) b\left(1+\frac{\sigma^{\prime}}{1+n}\right) \\
& , \text { for } z<a\left(1-\frac{\rho}{1+n}\right) b\left(1-\frac{\rho^{\prime}}{1+n}\right) \text { or } z>a\left(1+\frac{\sigma}{1+n}\right) b\left(1+\frac{\sigma^{\prime}}{1+n}\right)
\end{aligned}\right.
$$

where $P_{1}=\frac{a b \rho \rho^{1}}{(1+n)^{2}}, Q_{1}=\frac{a b}{1+n}\left[\left(1-\frac{\rho}{1+n}\right) \rho^{\prime}+\left(1-\frac{\rho^{\prime}}{1+n}\right) \rho\right], R_{1}=a b\left(1-\frac{\rho}{1+n}\right)\left(1-\frac{\rho^{\prime}}{1+n}\right)$, $P_{2}=\frac{a b \sigma \sigma^{1}}{(1+n)^{2}}, Q_{2}=-\frac{a b}{1+n}\left[\left(1+\frac{\sigma}{1+n}\right) \sigma^{\prime}+\left(1+\frac{\sigma^{\prime}}{1+n}\right) \sigma\right]$, and $R_{2}=a b\left(1+\frac{\sigma}{1+n}\right)\left(1+\frac{\sigma^{\prime}}{1+n}\right)$.

Graphically, the multiplication of two PDFNs is given in Figure 6.

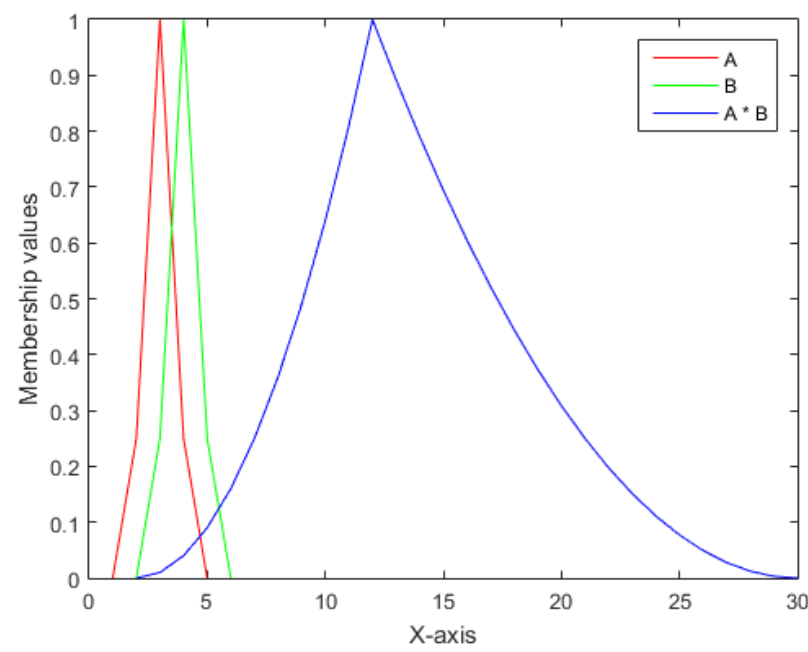

Figure 6. Multiplication of two parabolic dense fuzzy numbers.

\section{(v) Inverse of a Parabolic Dense Fuzzy Number}

Let $\widetilde{A}$ be a parabolic dense fuzzy number whose membership function is given in Equation (6). Then, the inverse of $\widetilde{A}$, i.e., $\widetilde{A}^{-1}=\left\langle a^{-1}\left(1+\frac{\sigma}{1+n}\right)^{-1}, a^{-1}, a^{-1}\left(1-\frac{\rho}{1+n}\right)^{-1}\right\rangle$, is also a parabolic dense fuzzy number whose membership function is

$$
\mu_{\widetilde{A}^{-1}}(z)=\left\{\begin{array}{l}
\left\{\frac{z a\left(1+\frac{\sigma}{1+n}\right)-1}{\frac{z+n}{1+n}}\right\}^{2}, \text { for } a^{-1}\left(1-\frac{\sigma}{1+n}\right)^{-1} \leq z \leq a^{-1} \\
\left\{\frac{1-a\left(1+\frac{\rho}{1+n}\right) z}{\frac{z a \rho}{1+n}}\right\}^{2}, \text { for } a^{-1} \leq z \leq a^{-1}\left(1+\frac{\rho}{1+n}\right)^{-1} \\
0, \text { for } z<a^{-1}\left(1-\frac{\sigma}{1+n}\right)^{-1} \text { or } z>a^{-1}\left(1+\frac{\rho}{1+n}\right)^{-1}
\end{array}\right.
$$

\section{Formulation of Crisp Model}

Here, we considered an inventory system of fishery culture. The fishery culture in a lake was mainly assumed for our proposed model. Here, for simplification of our study, all stages of fishery culture were not considered. We considered only from the stage where the fingerlings are brought from the market and fed to reach the ideal size for customer demand. Additional assumptions of the proposed model are given below. 
(i) We considered an additional cost for feeding the growing items.

(ii) Feeding cost depended on the weight of the items.

(iii) The growth function was approximated by a linear function.

(iv) We considered an idle cost for the growth period and cleaning period.

A schematic overview of the proposed model is given in Figure 7.

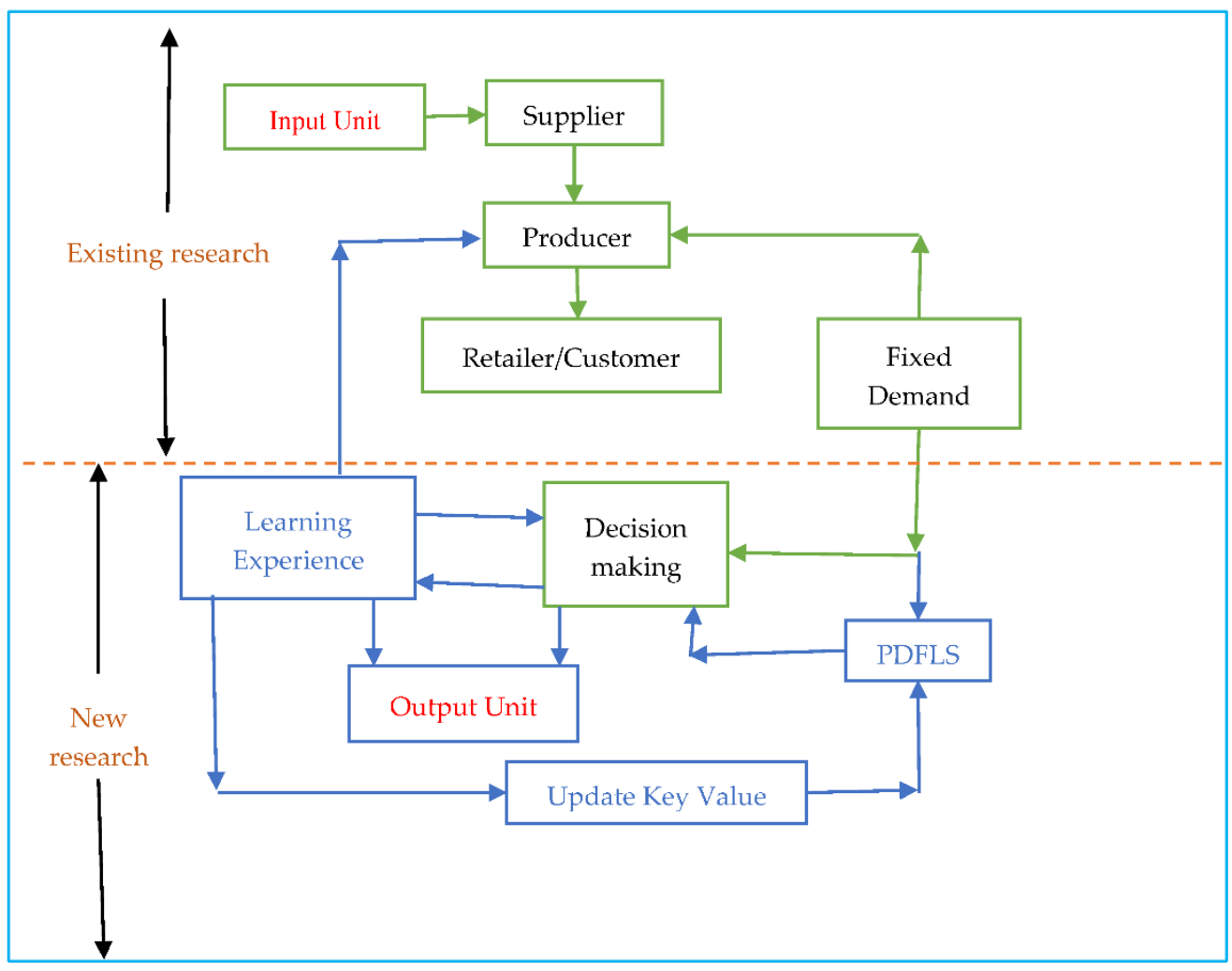

Figure 7. Schematic overview of the proposed model.

In Figure 8, $y$ is the number of purchased items and $g$ is the growth rate per fish per unit time. The initial approximate weight of each fingerling is $v_{0}$ and final approximate weight (at the time of selling) of each fish is $v_{1}$. At the beginning, $t_{1}$ is the time required to clean the pond, and then the fingerlings are grown up during the time $t_{2}$. After that, the fish are sold on the market during time $t_{3}$. According to this consideration, the following equations are obtained:

The total initial weight of fingerlings is $q_{0}=y v_{0}$.

The total final weight of the fishes is $q_{1}=y v_{1}$.

$$
\begin{gathered}
t_{2}=\frac{q_{1}-q_{0}}{y g}=\frac{v_{1}-v_{0}}{g} . \\
t_{3}=\frac{q_{1}}{d}=\frac{y v_{1}}{d} .
\end{gathered}
$$

Cycle time $T=t_{1}+t_{2}+t_{3}$. 
Inventory Level

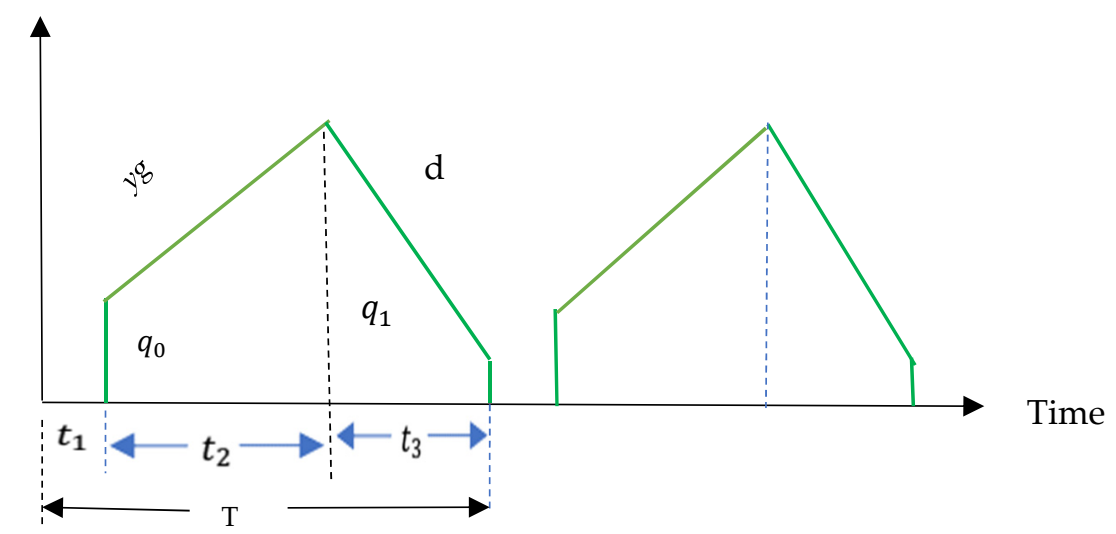

Figure 8. Diagram of the proposed model.

The main objective of this proposed model is to minimize the total cost $(Z)$ per cycle, which includes the purchasing cost, holding cost, food procurement cost (FPC), operational cost (OC), and natural idle cost (NIC).

$Z=$ purchasing $\cos t+$ holding $\cos t+$ food procurement $\cos t+$ operational $\cos t+$ natural idle $\cos t$,

where purchasing cost $=s y v_{0}=\frac{s v_{0} d t_{3}}{v_{1}}$, holding cost $=c_{2} T$, food procurement cost $=$ $\frac{f t_{2}\left(q_{1}-q_{0}\right)}{2}=\frac{f d t_{3}\left(v_{1}-v_{0}\right)^{2}}{2 g v_{1}}$, natural idle cost $=n_{\mathcal{c}}\left(t_{1}+t_{2}\right)$, and operational cost $=B$.

Putting these cost values into Equation (18) yields the total inventory cost per cycle as follows:

$$
\begin{aligned}
& Z=\frac{s v_{0} d t_{3}}{v_{1}}+c_{2} T+\frac{f d t_{3}\left(v_{1}-v_{0}\right)^{2}}{2 g v_{1}}+n_{c}\left(t_{1}+t_{2}\right)+B, \\
& =d\left\{\frac{s v_{0} t_{3}}{v_{1}}+\frac{f t_{3}\left(v_{1}-v_{0}\right)^{2}}{2 g v_{1}}\right\}+\left\{c_{2} T+n_{c}\left(t_{1}+t_{2}\right)+B\right\},
\end{aligned}
$$

where,

$$
\left\{\begin{array}{c}
\varphi=\frac{s v_{0} t_{3}}{v_{1}}+\frac{f t_{3}\left(v_{1}-v_{0}\right)^{2}}{2 g v_{1}} \\
\psi=c_{2} T+n_{c}\left(t_{1}+t_{2}\right)+B
\end{array}\right.
$$

Thus, the crisp problem is given by

$$
\text { Minimize } Z=d \varphi+\psi \text {, }
$$

subject to the conditions in Equations (13)-(17) and (20).

\section{Fuzzy Mathematical Model}

We know that the demand rate is a crucial part of any inventory problem, and it does not remain constant every time. Thus, we considered the demand rate as flexible throughout the proposed model, where it could be assumed as a parabolic dense fuzzy set. Hence, the objective function of the crisp model becomes

$$
\widetilde{Z}=\widetilde{d} \varphi+\psi,
$$

where $\varphi$ and $\psi$ are given in Equation (20).

The membership functions of the parabolic dense fuzzy demand rate with $n$ learning frequency are given by 


$$
\mu(\widetilde{d})=\left\{\begin{array}{l}
\left\{\frac{x-d\left(1-\frac{\rho}{1+n}\right)}{\frac{d \rho}{1+n}}\right\}^{2}, \text { for } d\left(1-\frac{\rho}{1+n}\right) \leq x \leq d \\
\left\{\frac{d\left(1+\frac{\sigma}{1+n}\right)-x}{\frac{d \sigma}{1+n}}\right\}^{2}, \text { for } d \leq x \leq d\left(1+\frac{\sigma}{1+n}\right) \\
0, \text { for } x<d\left(1-\frac{\rho}{1+n}\right) \text { or } x>d\left(1+\frac{\sigma}{1+n}\right)
\end{array}\right.
$$

The left and right $\alpha$-cuts of the above membership function are given by

$$
\left[\mu_{L}^{d}, \mu_{R}^{d}\right]=\left[d+\frac{d \rho(\sqrt{\alpha}-1)}{1+n}, d+\frac{d \sigma(1-\sqrt{\alpha})}{1+n}\right] .
$$

Then, the index value of the demand rate is given by

$$
\begin{gathered}
I(\widetilde{d})=\frac{1}{2 N} \sum_{n=1}^{N} \int_{\alpha=0}^{1}\left\{d+\frac{d \rho(\sqrt{\alpha}-1)}{1+n}+d+\frac{d \sigma(1-\sqrt{\alpha})}{1+n}\right\} d \alpha, \\
=\frac{1}{2 N} \sum_{n=1}^{N}\left\{2 d+\frac{2 d(\rho-\sigma)}{3(1+n)}+\frac{d(\sigma-\rho)}{1+n}\right\}, \\
=d+\frac{d(\sigma-\rho)}{6 N}\left\{\frac{1}{2}+\frac{1}{3}+\ldots+\frac{1}{N}\right\} .
\end{gathered}
$$

Again, the equation

$\mathrm{Z}=d \varphi+\psi$ can be rewritten as

$$
d=\frac{Z-\psi}{\varphi} .
$$

Substituting Equation (26) into Equation (23), we obtain the membership function of the objective function as follows:

$$
\mu(\widetilde{Z})=\left\{\begin{array}{c}
\left\{\frac{\frac{Z-\psi}{\varphi}-d\left(1-\frac{\rho}{1+n}\right)}{\frac{d \rho}{1+n}}\right\}^{2}, \text { for } d \varphi\left(1-\frac{\rho}{1+n}\right)+\psi \leq x \leq d \varphi+\psi \\
\left\{\frac{d\left(1+\frac{\sigma}{1+n}\right)-\frac{Z-\psi}{\varphi}}{\frac{d \sigma}{1+n}}\right\}^{2}, \text { for } d \varphi+\psi \leq x \leq d \varphi\left(1+\frac{\sigma}{1+n}\right)+\psi \\
0, \text { for } x<d \varphi\left(1-\frac{\rho}{1+n}\right)+\psi \text { or } x>d \varphi\left(1+\frac{\sigma}{1+n}\right)+\psi
\end{array}\right.
$$

The left and right $\alpha$-cuts of the above membership function are given by

$$
\left[\mu_{L}^{d}, \mu_{R}^{d}\right]=\left[d \varphi+\psi+\frac{d \rho \varphi(\sqrt{\alpha}-1)}{1+n}, d \varphi+\psi+\frac{d \sigma \varphi(1-\sqrt{\alpha})}{1+n}\right] .
$$

Then, the index value of the fuzzy objective function is given by

$$
\begin{gathered}
I(\widetilde{Z})=\frac{1}{2 N} \sum_{n=1}^{N} \int_{\alpha=0}^{1}\left\{d \varphi+\psi+\frac{d \rho \varphi(\sqrt{\alpha}-1)}{1+n}+d \varphi+\psi+\frac{d \sigma \varphi(1-\sqrt{\alpha})}{1+n}\right\} d \alpha, \\
=\frac{1}{2 N} \sum_{n=1}^{N}\left\{2(d \varphi+\psi)+\frac{2 d \varphi(\rho-\sigma)}{3(1+n)}+\frac{d \varphi(\sigma-\rho)}{1+n}\right\}, \\
=d \varphi+\psi+\frac{d \varphi(\sigma-\rho)}{6 N}\left\{\frac{1}{2}+\frac{1}{3}+\ldots+\frac{1}{N}\right\} .
\end{gathered}
$$

Thus, the problem under a parabolic dense fuzzy approach becomes

$$
\left\{\begin{array}{c}
\text { Maximize } I(\widetilde{Z})=d \varphi+\psi+\frac{d \varphi(\sigma-\rho)}{6 N}\left\{\frac{1}{2}+\frac{1}{3}+\ldots+\frac{1}{N}\right\} \\
I(\widetilde{d})=d+\frac{d(\sigma-\rho)}{6 N}\left\{\frac{1}{2}+\frac{1}{3}+\ldots+\frac{1}{N}\right\} \\
\text { Subject to condition (13)-(17) and (20) }
\end{array}\right.
$$


Next, we considered the demand rate as a parabolic dense fuzzy lock set, where the membership function of the demand rate becomes

$$
\mu(\widetilde{d})=\left\{\begin{aligned}
& \left\{\frac{x-d\left(1-\rho\left(\frac{1}{l_{1}}-\frac{1}{1+n}\right)\right)}{d \rho\left(\frac{1}{l_{1}}-\frac{1}{1+n}\right)}\right\}^{2}, \text { for } d\left(1-\rho\left(\frac{1}{l_{1}}-\frac{1}{1+n}\right)\right) \leq x \leq d \\
& \left\{\frac{d\left(1+\sigma\left(\frac{1}{l_{2}}-\frac{1}{1+n}\right)\right)-x}{d \sigma\left(\frac{1}{l_{2}}-\frac{1}{1+n}\right)}\right\}^{2}, \text { for } d \leq x \leq d\left(1+\sigma\left(\frac{1}{l_{2}}-\frac{1}{1+n}\right)\right) \\
& 0, \text { for } x<d\left(1-\rho\left(\frac{1}{l_{1}}-\frac{1}{1+n}\right)\right) \text { or } x>d\left(1+\sigma\left(\frac{1}{l_{2}}-\frac{1}{1+n}\right)\right)
\end{aligned}\right.
$$

The left and right $\alpha$-cuts of the above membership function are given by

$$
\left[\mu_{L}^{d}, \mu_{R}^{d}\right]=\left[d+d \rho(\sqrt{\alpha}-1)\left(\frac{1}{l_{1}}-\frac{1}{1+n}\right), d+d \sigma(1-\sqrt{\alpha})\left(\frac{1}{l_{2}}-\frac{1}{1+n}\right)\right] .
$$

Thus, the index value of demand rate is given by

$$
\begin{aligned}
I(\widetilde{d}) & =\frac{1}{2 N} \sum_{n=1}^{N} \int_{\alpha=0}^{1}\left\{\mu_{L}^{d}+\mu_{R}^{d}\right\} d \alpha, \\
& =\frac{1}{2 N} \sum_{n=1}^{N} \int_{\alpha=0}^{1}\left\{d+d \rho(\sqrt{\alpha}-1)\left(\frac{1}{l_{1}}-\frac{1}{1+n}\right)+d+d \sigma(1-\sqrt{\alpha})\left(\frac{1}{l_{2}}-\frac{1}{1+n}\right)\right\} d \alpha, \\
& =\frac{1}{2 N} \sum_{n=1}^{N}\left\{2 d+\frac{2 d \sigma}{3}\left(\frac{1}{l_{2}}-\frac{1}{1+n}\right)-\frac{2 d \rho}{3}\left(\frac{1}{l_{1}}-\frac{1}{1+n}\right)\right\}, \\
& =d+\frac{d}{3}\left(\frac{\sigma}{l_{2}}-\frac{\rho}{l_{1}}\right)+\frac{d(\rho-\sigma)}{3 N}\left\{\frac{1}{2}+\frac{1}{3}+\ldots+\frac{1}{N}\right\} .
\end{aligned}
$$

Then, substituting Equation (26) into Equation (31), we obtain the membership function of the fuzzy objective function as given below.

$$
\mu(\widetilde{Z})=\left\{\begin{array}{l}
\left\{\frac{\frac{z-\psi}{\varphi}-d\left(1-\rho\left(\frac{1}{l_{1}}-\frac{1}{1+n}\right)\right)}{d \rho\left(\frac{1}{l_{1}}-\frac{1}{1+n}\right)}\right\}^{2}, \text { for } d \varphi\left(1-\rho\left(\frac{1}{l_{1}}-\frac{1}{1+n}\right)\right)+\psi \leq z \leq d \varphi+\psi \\
\left\{\frac{d\left(1+\sigma\left(\frac{1}{l_{2}}-\frac{1}{1+n}\right)\right)-\frac{z-\psi}{\varphi}}{d \sigma\left(\frac{1}{l_{2}}-\frac{1}{1+n}\right)}\right\}^{2}, \text { for } d \varphi+\psi \leq z \leq d \varphi\left(1+\sigma\left(\frac{1}{l_{2}}-\frac{1}{1+n}\right)\right)+\psi \\
0, \text { for } x<d \varphi\left(1-\rho\left(\frac{1}{l_{1}}-\frac{1}{1+n}\right)\right)+\psi \text { or } x>d \varphi\left(1+\sigma\left(\frac{1}{l_{2}}-\frac{1}{1+n}\right)\right)+\psi
\end{array}\right.
$$

The left and right $\alpha$-cuts of the above membership function are given by

$$
\left[\mu_{L}^{d}, \mu_{R}^{d}\right]=\left[d \varphi+\psi+d \varphi \rho(\sqrt{\alpha}-1)\left(\frac{1}{l_{1}}-\frac{1}{1+n}\right), d \varphi+\psi+d \varphi \sigma(1-\sqrt{\alpha})\left(\frac{1}{l_{2}}-\frac{1}{1+n}\right)\right] .
$$

Then, the index value of the fuzzy objective function is given by

$$
\begin{array}{r}
I(\widetilde{Z})=\frac{1}{2 N} \sum_{n=1}^{N} \int_{\alpha=0}^{1}\left\{d \varphi+\psi+d \varphi \rho(\sqrt{\alpha}-1)\left(\frac{1}{l_{1}}-\frac{1}{1+n}\right), d \varphi+\psi\right. \\
\left.\quad+d \varphi \sigma(1-\sqrt{\alpha})\left(\frac{1}{l_{2}}-\frac{1}{1+n}\right)\right\} d \alpha, \\
=\frac{1}{2 N} \sum_{n=1}^{N}\left\{2(d \varphi+\psi)-\frac{2 d \varphi \rho}{3}\left(\frac{1}{l_{1}}-\frac{1}{1+n}\right)+\frac{2 d \varphi \sigma}{3}\left(\frac{1}{l_{2}}-\frac{1}{1+n}\right)\right\}, \\
=d \varphi+\psi+\frac{d \varphi}{3}\left(\frac{\sigma}{l_{2}}-\frac{\rho}{l_{1}}\right)+\frac{d \varphi(\rho-\sigma)}{3 N}\left\{\frac{1}{2}+\frac{1}{3}+\ldots+\frac{1}{N}\right\} .
\end{array}
$$

Thus, the problem under a PDFLS approach becomes

$$
\left\{\begin{array}{c}
\text { Maximize } I(\widetilde{Z})=d \varphi+\psi+\frac{d \varphi}{3}\left(\frac{\sigma}{l_{2}}-\frac{\rho}{l_{1}}\right)+\frac{d \varphi(\rho-\sigma)}{3 N}\left\{\frac{1}{2}+\frac{1}{3}+\ldots+\frac{1}{N}\right\} \\
I(\widetilde{d})=d+\frac{d}{3}\left(\frac{\sigma}{l_{2}}-\frac{\rho}{l_{1}}\right)+\frac{d(\rho-\sigma)}{3 N}\left\{\frac{1}{2}+\frac{1}{3}+\ldots+\frac{1}{N}\right\} \\
\text { Subject to condition (13)-(17) and (20) }
\end{array}\right.
$$


If we substitute $l_{1}=l_{2}=1$ and $N \rightarrow \infty$ into Equation (37), then the TDFLS problem becomes a general fuzzy problem.

$$
\left\{\begin{array}{c}
\text { Maximize I }(\widetilde{Z})=d \varphi+\psi+\frac{d \varphi}{3}(\sigma-\rho)+\frac{d \varphi(\rho-\sigma)}{3 N}\left\{\frac{1}{2}+\frac{1}{3}+\ldots+\frac{1}{N}\right\} \\
I(\widetilde{d})=d+\frac{d}{3}(\sigma-\rho)+\frac{d(\rho-\sigma)}{3 N}\left\{\frac{1}{2}+\frac{1}{3}+\ldots+\frac{1}{N}\right\} \\
\text { Subject to condition (13)-(17) and (20) }
\end{array}\right.
$$

\subsection{Rules of Finding Key Values of the Fuzzy Locks}

Every fuzzy parameter has two bounds, namely, an upper bound ( $b^{U}$, if known) and a lower bound $\left(b^{L}\right.$, if known) or both. For a single key, if the upper bound is known, then the index value of $\widetilde{b}$ is given by $I(\widetilde{b}) \leq b^{U}$, which implies $k \geq \frac{b(\sigma-\rho)}{4\left(b^{U}-b\right)}$. If the lower bound is given, then $I(\widetilde{b}) \geq b^{L}$ implies $k \geq \frac{b(\rho-\sigma)}{4\left(b-b^{L}\right)}$. For double keys, the index value of $\widetilde{A}$ is given by

$$
I(\widetilde{B})=\frac{1}{2 N} \sum_{n=0}^{N} \int_{0}^{1}\left\{\mu_{L}^{B}+\mu_{R}^{B}\right\} d \alpha=\frac{b}{2}\left(1-\frac{\rho}{2 l_{1}}\right)+\frac{b}{2}\left(1+\frac{\sigma}{2 l_{2}}\right) .
$$

Thus,

$$
\frac{b}{2}\left(1-\frac{\rho}{2 l_{1}}\right) \geq b^{L} \Rightarrow l_{1} \geq \frac{b \rho}{2\left(b-2 b^{L}\right)} \text { and } \frac{b}{2}\left(1+\frac{\sigma}{2 l_{2}}\right) \leq b^{U} \Rightarrow l_{2} \geq \frac{b \sigma}{2\left(2 b^{U}-b\right)} .
$$

\subsection{Solution Algorithm}

The solution algorithm of our proposed model is given below. Input: Values of all parameters $\left(d, g, v_{0}, v_{1}, t_{1}, s, f, c_{2}, n_{c}, B\right.$, $l_{1}, l_{2}, \rho$ and $\left.\sigma\right)$.

Output: The optimum inventory cost of the proposed model.

Step 1. Solve the problem in a crisp sense first using Equation (21).

Step 2. Consider demand as a parabolic dense fuzzy number.

Step 3. Find the index value of the inventory cost function in PDFS approach using Equation (30).

Step 4. Consider demand as a parabolic dense fuzzy lock set.

Step 5. Find the corresponding inventory cost using Equation (37).

Step 6. Compare among crisp, general fuzzy, PDFS, and PDFLS results and obtain the minimum inventory cost of the proposed model.

Step 7. End.

\section{A Real Case Study}

We visited the West Bengal fisheries corporation Ltd. situated in Sector V, Salt Lake City, Kolkata, West Bengal 700091, India ((latitude, longitude $\left.)=\left(22.5691^{\circ} \mathrm{N}, 88.4337^{\circ} \mathrm{E}\right)\right)$ on 10 December 2020. After a long discussion with the manager Mr. Mukherjee, we knew that they cleaned the lake properly at the beginning of each cycle. After that, the fingerlings were stocked and fed in the lake until the fingerlings acquired the ideal size for selling. We collected some valuable data related to our proposed model: $d=4,000,000 \mathrm{~g} /$ year, $g=912.5 \mathrm{~g} /$ year, $v_{0}=100 \mathrm{~g}$,

$$
v_{1}=600 \mathrm{~g} \text {, }
$$

$t_{1}=0.019$ year, $s=\$ 0.01 \mathrm{~g}, f=\$ 0.8 / \mathrm{g} /$ year, $c_{2}=\$ 100 /$ year, $n_{c}=\$ 40 /$ year, $B=\$ 80 /$ year, $\rho=0.4$, and $\sigma=0.2$. The upper bound and lower bound of the demand rate were set to $d_{0}^{L}=3,600,000$ and $d_{0}^{U}=4,000,000$. Utilising the above rule, the key values of the demand rate were obtained as follows: 


$$
\left[\begin{array}{l}
l_{1} \\
l_{2}
\end{array}\right] \geq\left[\begin{array}{c}
\frac{d \rho}{2\left(d-2 d^{L}\right)} \\
\frac{d \sigma}{2\left(2 d^{U}-d\right)}
\end{array}\right]=\left[\begin{array}{c}
\frac{4,000,000 \times 0.4}{2(4,000,000-2 \times 3,600,000)} \\
\frac{4,000,000 \times 0.2}{2(2 \times 4,400,000-4,000,000)}
\end{array}\right]=\left[\begin{array}{c}
-0.25 \\
0.083
\end{array}\right]
$$

Satisfying the above condition, let $l_{1}=0.38$ and $l_{2}=0.25$; accordingly, the optimal solutions of the crisp model and several fuzzy models are given in Table 2.

Table 2. Numerical result of the proposed model.

\begin{tabular}{|c|c|c|c|c|c|c|}
\hline Approach & $N^{*}$ & Time $\left(t_{2}^{*}\right)$ & Time $\left(t_{3}^{*}\right)$ & Ordered Items $\left(y^{*}\right)$ & Total Cost $\left(Z^{*}\right)$ & $\frac{Z^{*}-Z_{\text {Crisp }}^{*}}{Z_{\text {Crisp }}^{*}} \times \mathbf{1 0 0}$ \\
\hline Crisp & - & 0.5479 & 0.4330 & 2887 & $319,476.8$ & - \\
\hline General Fuzzy & - & 0.5479 & 0.4330 & 2790 & $308,834.3$ & -3.33 \\
\hline \multirow{3}{*}{ Parabolic dense fuzzy } & 1 & 0.5479 & 0.4327 & 2838 & $314,155.5$ & -1.66 \\
\hline & 2 & 0.5479 & 0.4321 & 2846 & $315,042.4$ & -1.39 \\
\hline & 3 & 0.5479 & 0.4318 & 2852 & $315,633.7$ & -1.20 \\
\hline \multirow{3}{*}{ PDFLS } & 1 & 05479 & 0.4330 & 2740 & $303,233.0$ & -5.08 \\
\hline & 2 & 0.5479 & 0.4331 & 2724 & $301,459.3$ & -5.64 \\
\hline & 3 & 0.5479 & 0.4330 & 2713 & $300,276.8$ & -6.00 \\
\hline
\end{tabular}

Table 2 shows that the total inventory cost of the proposed model is $\$ 319,476.8$ in the crisp case. For a general fuzzy environment, the inventory cost is $\$ 308,834$.3. In a parabolic dense fuzzy environment, the minimum total inventory cost is $\$ 314,155.5$ for learning experience $N=1$, whereas, for TDFLS, the minimum total inventory cost is $\$ 300,276.8$ with a growing period of 0.5479 years $\approx 200$ days, a selling period of 0.4327 years $\approx 158$ days, and 2838 ordered items for learning experience $N=3$. From Table 2, it is also clear that, in the general fuzzy model, the total inventory cost decreases by $3.33 \%$. In the parabolic dense fuzzy model, the total inventory cost decreases by $1.66 \%$, whereas, in the PDFLS model, the total inventory cost decreases by $6.00 \%$. Thus, TDFLS gave the minimum inventory cost of the proposed model.

\section{Sensitivity Analysis}

From Table 2, we can see that parabolic dense fuzzy lock set gave the optimum value of the proposed model. Hence, we conducted a sensitivity analysis over the parabolic dense fuzzy model. We changed each parameter $\left(v_{0}, v_{1}, s, f, c_{2}, n_{c}, \rho\right.$, and $\left.\sigma\right)$ from $-30 \%$ to $+30 \%$, and the results are given in Table 3. Furthermore, a graphical illustration of Table 3 is presented in Section 7.

Table 3. Sensitivity analysis of the parabolic dense fuzzy model.

\begin{tabular}{|c|c|c|c|c|c|c|}
\hline Parameter & $\%$ Change & Time $\left(t_{2}^{*}\right)$ & Time $\left(t_{3}^{*}\right)$ & Ordered Items $\left(y^{*}\right)$ & Total Cost $\left(Z^{*}\right)$ & $\frac{Z^{*}-Z_{\text {Crisp }}^{*}}{Z_{\text {Crisp }}^{*}} \times 100$ \\
\hline \multirow{4}{*}{$v_{0}$} & +30 & 0.5151 & 0.4659 & 2919 & $286,691.8$ & -10.26 \\
\hline & +10 & 0.5369 & 0.4440 & 2782 & $296,074.7$ & -7.32 \\
\hline & -10 & 0.5589 & 0.4220 & 2644 & $304,128.4$ & -4.80 \\
\hline & -30 & 0.5808 & 0.4002 & 2507 & $310,708.3$ & -2.74 \\
\hline \multirow{4}{*}{$v_{1}$} & +30 & 0.7452 & 0.2358 & 1136 & $231,708.0$ & -27.47 \\
\hline & +10 & 0.6137 & 0.3673 & 2092 & $289,909.2$ & -9.25 \\
\hline & -10 & 0.4822 & 0.4988 & 3472 & $298,384.2$ & -6.60 \\
\hline & -30 & 0.3507 & 0.6303 & 5642 & $259,093.1$ & -18.90 \\
\hline \multirow{4}{*}{$S$} & +30 & 0.5479 & 0.4330 & 2713 & $301,090.8$ & -5.75 \\
\hline & +10 & 0.5479 & 0.4330 & 2713 & $300,548.1$ & -5.92 \\
\hline & -10 & 0.5479 & 0.4330 & 2713 & $300,005.4$ & -6.09 \\
\hline & -30 & 0.5479 & 0.4330 & 2713 & $299,462.7$ & -6.26 \\
\hline
\end{tabular}


Table 3. Cont.

\begin{tabular}{|c|c|c|c|c|c|c|}
\hline Parameter & $\%$ Change & Time $\left(t_{2}^{*}\right)$ & Time $\left(t_{3}^{*}\right)$ & Ordered Items $\left(y^{*}\right)$ & Total Cost $\left(Z^{*}\right)$ & $\frac{Z^{*}-Z_{\text {Crisp }}^{*}}{Z_{\text {Crisp }}^{*}} \times 100$ \\
\hline \multirow{4}{*}{$f$} & +30 & 0.5479 & 0.4330 & 2713 & $389,485.0$ & +21.91 \\
\hline & +10 & 0.5479 & 0.4330 & 2713 & $330,012.8$ & +3.29 \\
\hline & -10 & 0.5479 & 0.4330 & 2713 & $270,540.7$ & -15.31 \\
\hline & -30 & 0.5479 & 0.4330 & 2713 & $211,068.6$ & -33.93 \\
\hline \multirow{4}{*}{$c_{2}$} & +30 & 0.5479 & 0.4330 & 2713 & $300,306.8$ & -6.00 \\
\hline & +10 & 0.5479 & 0.4330 & 2713 & $300,286.3$ & -6.00 \\
\hline & -10 & 0.5479 & 0.4330 & 2713 & $300,266.8$ & -6.01 \\
\hline & -30 & 0.5479 & 0.4330 & 2713 & $300,246.8$ & -6.01 \\
\hline \multirow{4}{*}{$n_{c}$} & +30 & 0.5479 & 0.4330 & 2713 & $300,283.6$ & -6.00 \\
\hline & +10 & 0.5479 & 0.4330 & 2713 & $300,279.0$ & -6.00 \\
\hline & -10 & 0.5479 & 0.4330 & 2713 & $300,274.5$ & -6.01 \\
\hline & -30 & 0.5479 & 0.4330 & 2713 & $300,270.0$ & -6.01 \\
\hline \multirow{4}{*}{$\rho$} & +30 & 0.5479 & 0.4330 & 2451 & $271,280.7$ & -15.08 \\
\hline & +10 & 0.5479 & 0.4330 & 2626 & $290,611.4$ & -9.03 \\
\hline & -10 & 0.5479 & 0.4330 & 2800 & $309,942.1$ & -2.98 \\
\hline & -30 & 0.5479 & 0.4330 & 2975 & $329,272.8$ & +3.06 \\
\hline \multirow{4}{*}{$\sigma$} & +30 & 0.5479 & 0.4330 & 2923 & $323,512.8$ & +1.26 \\
\hline & +10 & 0.5479 & 0.4330 & 2783 & $308,022.1$ & -3.58 \\
\hline & -10 & 0.5479 & 0.4330 & 2643 & $292,531.4$ & -8.43 \\
\hline & -30 & 0.5479 & 0.4330 & 2503 & $277,040.7$ & -13.28 \\
\hline
\end{tabular}

\section{Graphical Illustration}

In this section, we provide several graphs (Figures 9-13) to illustrate the numerical results in Table 2 and the sensitivity analysis results in Table 3. The figures are given below.

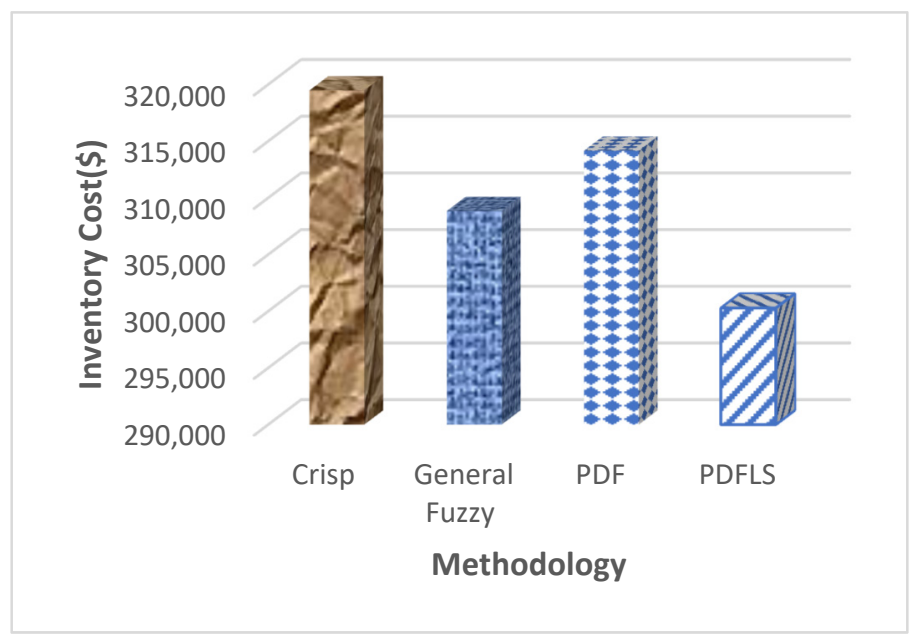

Figure 9. Inventory cost vs. methodology.

Figure 9 shows that the inventory cost obtains the minimum value in a PDFLS environment, whereas it obtains the maximum in a crisp sense. Moreover, the inventory cost for the general fuzzy approach assumes a lower value than the parabolic dense fuzzy approach. Thus, by applying the parabolic dense fuzzy lock set approach, the inventory practitioner can minimize the total inventory cost. 


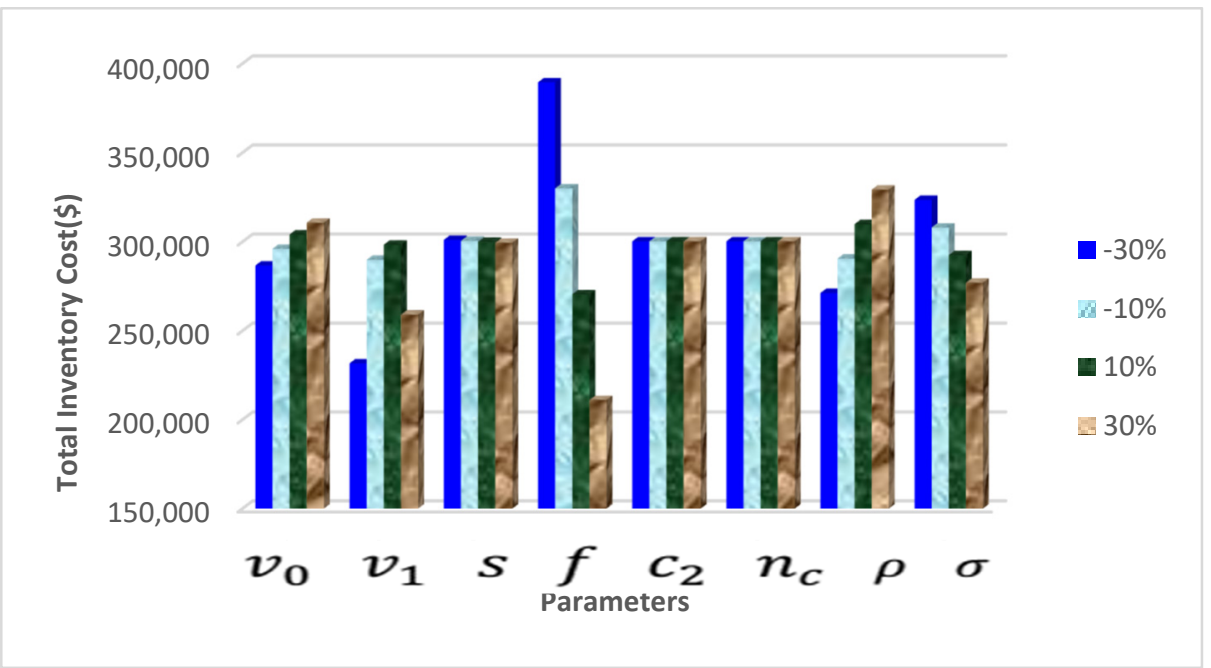

Figure 10. Sensitivity analysis of parameters.

Figure 10 shows that the approximated weight of fingerlings $\left(v_{0}\right)$ and deviation parameter $(\rho, \sigma)$ were not very sensitive, whereas the price of fingerlings $(s)$, holding cost $\left(c_{2}\right)$, and natural idle cost $\left(n_{c}\right)$ were insensitive within this variation. However, the feeding cost $(f)$ and approximated weight of fish at the time of selling $\left(v_{1}\right)$ were highly sensitive parameters for this system. Thus, to minimize the inventory cost, these two parameters $\left(f, v_{1}\right)$ should be handled carefully.

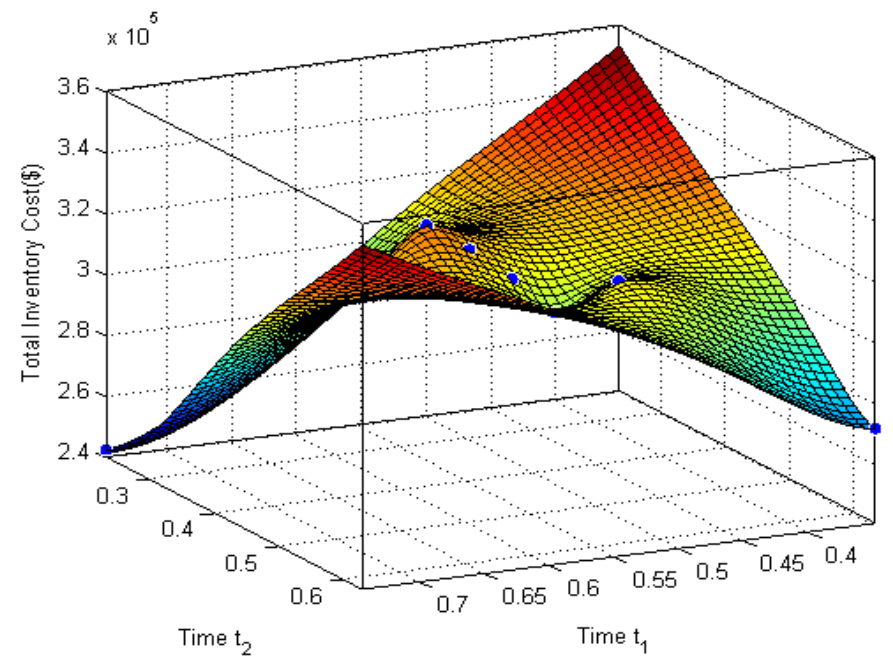

Figure 11. Total inventory cost vs. time $t_{1}$ and time $t_{2}$.

Figure 11 shows how the total inventory cost depends on time $t_{1}$ and time $t_{2}$. From this surface figure, it is clear that, when feeding time $t_{1}$ increases and selling period $t_{2}$ decreases, the inventory cost obtains the minimum value. Moreover, when feeding time $t_{1}$ decreases and selling period $t_{2}$ increases, the inventory cost decreases. On the other hand, when both $\left(t_{1}, t_{2}\right)$ increase or decrease together, the inventory cost also increases. 


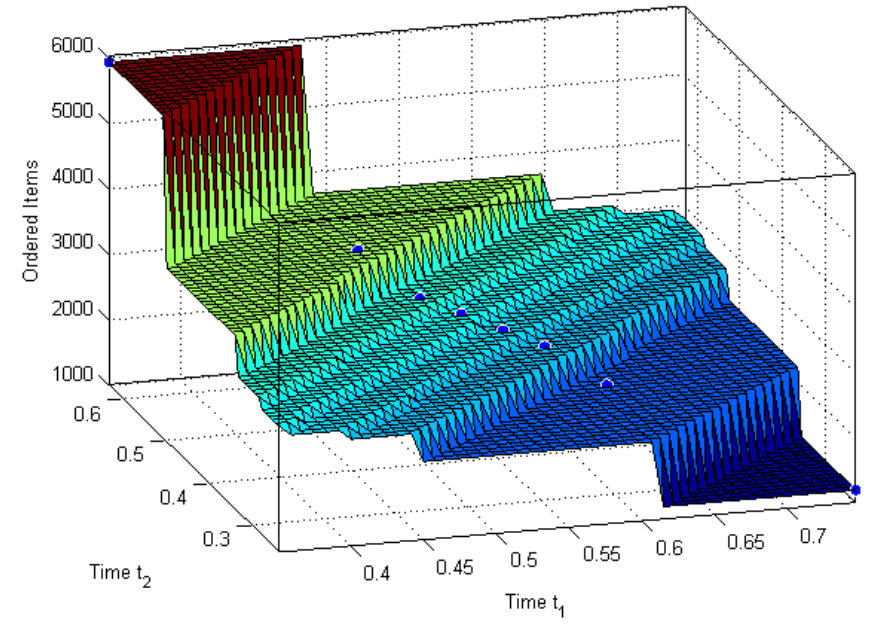

Figure 12. Ordered items vs. time $t_{1}$ and time $t_{2}$.

Figure 12 represents the relationship among ordered items, feeding time $\left(t_{1}\right)$, and selling period $\left(t_{2}\right)$. From this figure, it is clear that ordered items obtain the maximum value when the feeding time is minimum and the selling period is maximum. Furthermore, ordered items obtain the minimum value when the feeding time is maximum and the selling period is minimum.

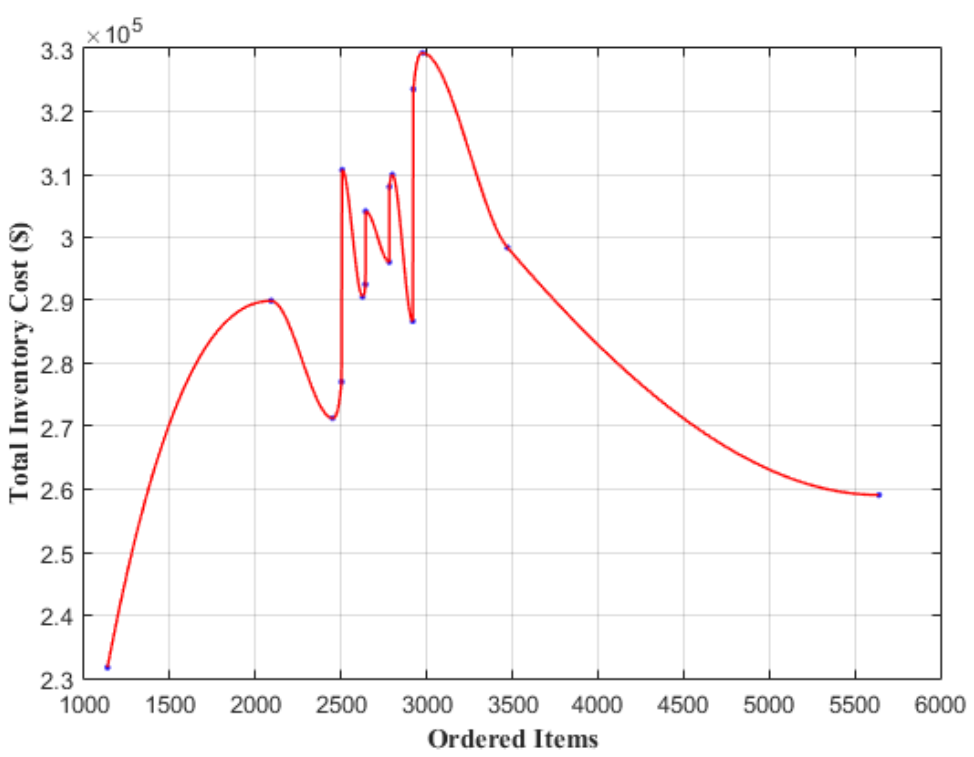

Figure 13. Total inventory cost vs. ordered items.

Figure 13 illustrates the relationship between total inventory cost and ordered items. In this figure, a smooth spline curve was drawn using the data of $y^{*}$ and $Z^{*}$ from Table 3 . From the above figure, it is clear that total inventory cost does not monotonically increase with respect to ordered items. Therefore, from this graphical illustration, we can easily predict the value of ordered items when the total inventory cost is supposed to obtain a minimum value. 


\section{Managerial Insights}

In this article, we studied some basic arithmetic operations over parabolic dense fuzzy numbers. We also defined the parabolic dense fuzzy lock set and its application in inventory management. An EOQ model of fishery culture was considered as a case study. We evaluated several decision variables such as the growing period (year), selling period (year), and the number of items ordered in each period. A decision maker can solve/tackle any complex situation of an entire industry with proper insight. The basic managerial insights of our proposed model are given below.

(i) Learning experience is a crucial part of any inventory problem to optimize the inventory cost.

(ii) The decision maker can optimize the inventory cost by utilizing proper key values.

(iii) The less qualified decision maker can also minimize the cost using the PDFLS approach.

(iv) The PDFLS approach gives a finer optimum than the parabolic dense set approach.

\section{Conclusions}

In this paper, we defined the parabolic dense fuzzy number with some basic arithmetic operations. Here, we developed an EOQ model for fishery culture using a real case study. We assumed that a linear function could estimate the growth rate of fish. The proposed EOQ inventory model studied the fishery culture that fed fingerlings until they were ready for sale with an ideal weight for the customer. Here, natural idle cost was considered during feeding and cleaning time. The main aim of this work was to find the number of ordering items such that the total inventory cost was minimized. In the fuzzy mathematical model, the demand rate was considered as a parabolic dense fuzzy lock set. The numerical result revealed that the parabolic dense fuzzy lock model obtained the minimum cost compared with the crisp and other fuzzy models.

This model also had some limitations. This model is not applicable to experience-free enterprises or newly set up inventory systems. In this approach, adequate manpower is required to collect more information about the model.

For future research, researchers may use the PDFLS concept in various inventory problems such as supply chain management problems, transportation problems, and trade credit problems. The PDFLS concept may be incorporate into a trapezoidal fuzzy number, pentagonal fuzzy number, hexagonal fuzzy number, heptagonal fuzzy number, etc. for a more realistic approach.

Author Contributions: Conceptualization, S.K.D., M.P. and S.P.M.; Formal analysis, S.M., S.K.D. and S.P.M.; Investigation, S.P.M.; Methodology, S.M.; Project administration, M.P.; Resources, S.M. and M.P.; Software, S.M.; Supervision, S.P.M.; Validation, S.K.D.; Visualization, S.K.D. and M.P. All authors have read and agreed to the published version of the manuscript.

Funding: This research received no external funding.

Institutional Review Board Statement: Not applicable.

Informed Consent Statement: Not applicable.

Data Availability Statement: Not applicable.

Conflicts of Interest: The authors declare no conflict of interest.

\section{Notations}

The notations used in this model are given below. 


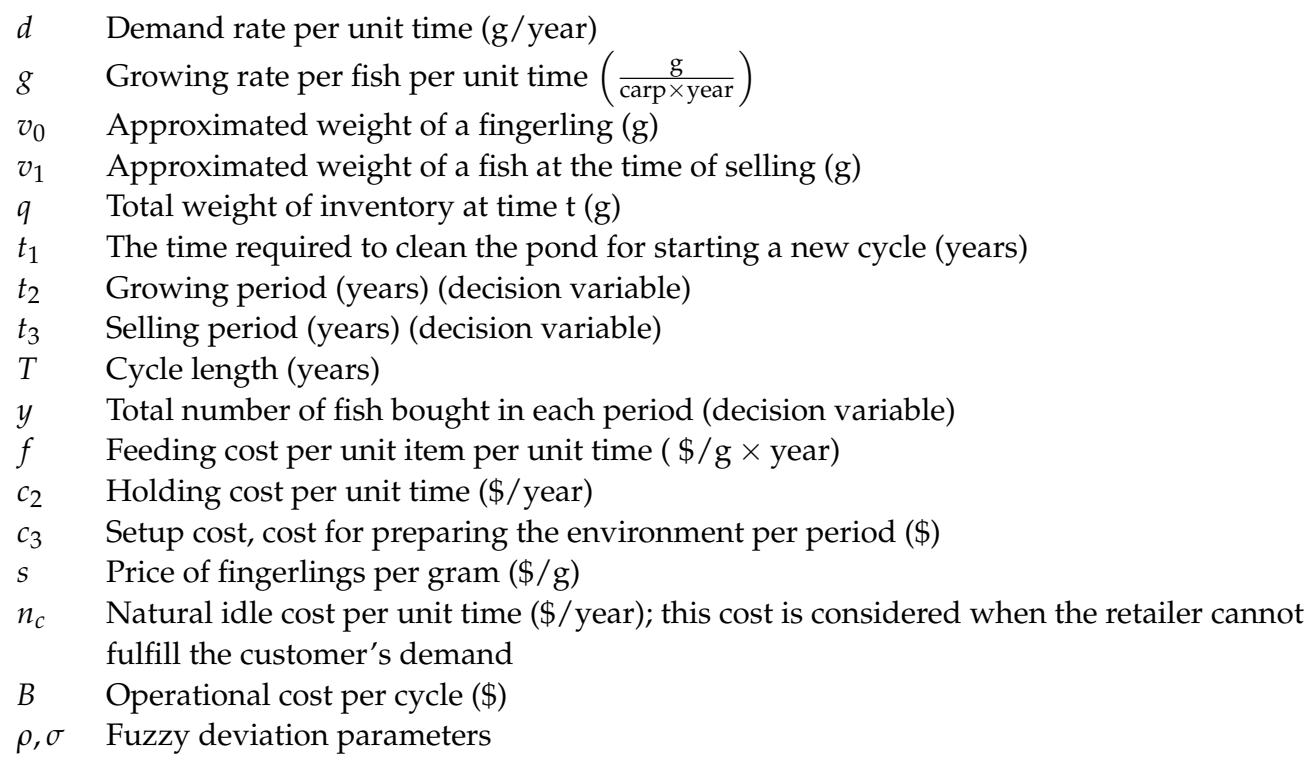

\section{References}

1. Zadeh, L.A. Fuzzy sets. Inf. Control 1965, 8, 338-356. [CrossRef]

2. Bellman, R.E.; Zadeh, L.A. Decision making in a fuzzy environment. Manag. Sci. 1970, 17, 141-164. [CrossRef]

3. Dubois, D.; Prade, H. Operations on fuzzy numbers. Int. J. Syst. Sci. 1978, 9, 613-626. [CrossRef]

4. Atanassov, K.T. Intuitionistic fuzzy sets. In Proceedings of the VII ITKR's Session, Sofia, Bulgaria, $20-23$ June 1983.

5. Kaufmann, A.; Gupta, M.M. Introduction of Fuzzy Arithmetic Theory and Applications; Van Nostrand Reinhold: New York, NY, USA, 1985.

6. Chutia, R.; Mahanta, S.; Baruah, H.K. An Alternative Method of Finding the Membership of a Fuzzy Number. Int. J. Latest Trends Comput. 2010, 1, 69-72.

7. De, S.K.; Beg, I. Triangular dense fuzzy sets and new defuzzication methods. J. Intell. Fuzzy Syst. 2016, 36, 469-477. [CrossRef]

8. De, S.K.; Mahata, G.C. Decision of a fuzzy inventory with fuzzy backorder model under cloudy fuzzy demand rate. Int. J. Appl. Comput. Math. 2016, 3, 2593-2609. [CrossRef]

9. De, S.K. Triangular Dense Fuzzy Lock Set. Soft Comput. 2017, 22, 7243-7254. [CrossRef]

10. Faritha, A.A.; Priya, G. Optimizing triangular parabolic fuzzy EOQ model with shortage using nearest interval approximation. Int. J. Future Revolut. Comput. Sci. Commun. Eng. 2017, 3, 92-96.

11. Garg, H.; Ansha. Arithmetic operations on generalized parabolic fuzzy numbers and its application. Proc. Natl. Acad. Sci. India Sect. A Phys. Sci. 2016, 88, 15-26. [CrossRef]

12. Maity, S.; Chakraborty, A.; De, S.K.; Mondal, S.P.; Alam, S. A comprehensive study of a backlogging EOQ model with non-linear heptagonal dense fuzzy environment. RAIRO-Oper. Res. 2020, 54, 267-286. [CrossRef]

13. Maity, S.; De, S.K.; Mondal, S.K. A Study of a Backorder EOQ Model for Cloud-Type Intuitionistic Dense Fuzzy Demand Rate. Int. J. Fuzzy Syst. 2019, 22, 201-211. [CrossRef]

14. Harris, F.W. Operations and Costs (Factory Management Series); A. W. Shaw Co.: Chicago, IL, USA, 1913; pp. 18-52.

15. Karlin, S. One Stage Inventory Model with Uncertainty, Studies in the Mathematical Theory of Inventory and Production; Stanford University Press: Stanford, CA, USA, 1958.

16. De, S.K. EOQ model with natural idle time and wrongly measured demand rate. Int. J. Inventory Control Manag. 2013, 3, 329-354.

17. Das, P.; De, S.K.; Sana, S.S. An EOQ model for time dependent backlogging over idle time: A step order fuzzy approach. Int. J. Appl. Comput. Math. 2014, 1, 171-185. [CrossRef]

18. De, S.K.; Goswami, A.; Sana, S.S. An interpolating by pass to Pareto optimality in intuitionistic fuzzy technique for an EOQ model with time sensitive backlogging. Appl. Math. Comput. 2014, 230, 664-674. [CrossRef]

19. Kazemi, N.; Olugu, E.U.; Salwa Hanim, A.-R.; Ghazilla, R.A.B.R. Development of a fuzzy economic order quantity model for imperfect quality items using the learning effect on fuzzy parameters. J. Intell. Fuzzy Syst. 2015, 28, 2377-2389. [CrossRef]

20. De, S.K.; Sana, S.S. The (p, q, r, l) model for stochastic demand under intuitionistic fuzzy aggregation with Bonferroni mean J. Intell. Manuf. 2016, 29, 1753-1771. [CrossRef]

21. Karmakar, S.; De, S.K.; Goswami, A. A pollution sensitive dense fuzzy economic production quantity model with cycle time dependent production rate. J. Clean. Prod. 2017, 154, 139-150. [CrossRef]

22. Karmakar, S.; De, S.K.; Goswami, A. A pollution sensitive remanufacturing model with waste items: Triangular dense fuzzy lock set approach. J. Clean. Prod. 2018, 187, 789-803. [CrossRef]

23. Maity, S.; De, S.K.; Pal, M. Two Decision Makers' Single Decision over a Back Order EOQ Model with Dense Fuzzy Demand Rate. Financ. Mark. 2018, 3, 1-11. 
24. Maity, S.; De, S.K.; Mondal, S.P. A Study of an EOQ Model under Lock Fuzzy Environment. Mathematics 2019, 7, 75. [CrossRef]

25. De, S.K.; Mahata, G.C. A cloudy fuzzy economic order quantity model for imperfect-quality items with allowable proportionate discounts. J. Ind. Eng. Int. 2019, 15, 571-583. [CrossRef]

26. Liang, R.; Wang, J.Q. A Linguistic Intuitionistic Cloud Decision Support Model with Sentiment Analysis for Product Selection in E-commerce. Int. J. Fuzzy Syst. 2019, 21, 963-977. [CrossRef]

27. Nobil, A.H.; Sedigh, A.H.A.; Cárdenas-Barrón, L.E. A Generalized Economic Order Quantity Inventory Model with Shortage: Case Study of a Poultry Farmer. Arab. J. Sci. Eng. 2018, 44, 2653-2663. [CrossRef]

28. Rezaei, J. Economic order quantity for growing items. Int. J. Prod. Econ. 2014, 155, 109-113. [CrossRef]

29. Zhang, Y.; Li, L.Y.; Tian, X.Q.; Feng, C. Inventory management research for growing items with carbon-constrained. In Proceedings of the Control Conference (CCC), IEEE 35th Chinese, Chengdu, China, 27-29 July 2016; pp. 9588-9593.

30. De, S.K.; Mahata, G.C.; Maity, S. Carbon emission sensitive deteriorating inventory model with trade credit under volumetric fuzzy system. Int. J. Intell. Syst. 2021, 1-28. [CrossRef]

31. Mahata, G.C.; De, S.K.; Maity, S.; Bhattacharya, K. Three-echelon supply chain model in an imperfect production system with inspection error, learning effect, and return policy under fuzzy environment. Int. J. Syst. Sci. Oper. Logist. 2021. [CrossRef]

32. Salameh, M.K.; Jaber, M.Y. Economic production quantity model for items with imperfect quality. Int. J. Prod. Econ. 2000, 64, 59-64. [CrossRef]

33. Sebatjane, M.; Adetunji, O. Economic order quantity model for growing items with imperfect quality. Oper. Res. Perspect. 2019, 6, 100088. [CrossRef]

34. Wee, H.M.; Yu, J.; Chen, M.C. Optimal inventory model for items with imperfect quality and shortage backordering. Omega 2007, 35, 7-11. [CrossRef]

35. Alfares, H.K.; Afzal, A.R. An Economic Order Quantity Model for Growing Items with Imperfect Quality and Shortages. Arab. J. Sci. Eng. 2021, 46, 1863-1875. [CrossRef]

36. Rana, K.; Sing, S.R.; Saxena, N.; Sana, S.S. Growing items inventory model for carbon emission under the permissible delay in payment with partially backlogging. Green Financ. 2021, 3, 153-174. [CrossRef]

37. Choudhury, M.; Mahata, G.C. Sustainable integrated and pricing decisions for two-echelon supplier-retailer supply chain of growing Items. RAIRO-Oper. Res. 2021. [CrossRef]

38. De-la-Cruz-Márquez, C.G.; Cárdenas-Barrón, L.E.; Mandal, B. An Inventory Model for Growing Items with Imperfect Quality When the Demand Is Price Sensitive under Carbon Emissions and Shortages. Math. Probl. Eng. 2021, 2021, 6649048. [CrossRef]

39. Gharaei, A.; Almehdawe, E. Optimal sustainable order quantities for growing items. J. Clean. Prod. 2021, 307, 127216. [CrossRef]

40. Mittal, M.; Sharma, M. Economic Ordering Policies for Growing Items (Poultry) with Trade-Credit Financing. Int. J. Appl. Comput. Math. 2021, 7, 39. [CrossRef] 